\title{
Estudio de factores de riesgo para el desarrollo de pie diabético: Campaña 1N
}

\section{A study of risk factors in the development of diabetic foot: 1N Campaign}

\author{
Claudia Marcela Issa ${ }^{1,2}$, Gabriela Verónica Carro' ${ }^{1,3}$, Rubén Nicolás Saurral ${ }^{1,4}$, Elizabet Gladys Méndez ${ }^{1,5}$, \\ Claudio Fabián Dituro ${ }^{1,6}$, Javier Aníbal Vilte' ${ }^{1,7}$, Cristina Varela ${ }^{1,8}$, Raúl Alejandro David ${ }^{1,9}$, Érica Lorena Witman ${ }^{1,10}$, \\ Julio César Torres ${ }^{1,11}$, Rocío Zabala ${ }^{1,12}$, Gabriela Silvana Illuminati ${ }^{1,13}$, María Alejandra Casen ${ }^{1,14}$, Pablo Alterini ${ }^{1,15}$, \\ María Victoria Dicatarina Losada ${ }^{1,16}$, Sandra Aixa Kremer Sendros ${ }^{1,17}$, Florencia Cuto ${ }^{1,18}$, Florencia Badías ${ }^{1,19}$, \\ José Daniel Braver ${ }^{1,20}$, Luisa Mabel Carrió ${ }^{1,21}$, Lucía Belén Jiménez ${ }^{22}$, Jaqueline Norma Castillo ${ }^{23}$, \\ Javier Mauricio Farías ${ }^{24}$
}

\section{RESUMEN}

Introducción: el examen del pie es fundamental en pacientes con diabetes mellitus (DM). La correcta evaluación del pie en el paciente que concurre a control diabetológico es clave para establecer factores de riesgo para el desarrollo de úlceras, detectar lesiones, tomar medidas preventivas, realizar una derivación temprana y educar en cuidados del pie.

Objetivos: en este estudio se examinaron los pies de personas con DM durante la Campaña de Concientización y Prevención del Pie Diabético el $1^{\circ}$ de noviembre de 2019 con el fin de evaluar la presencia de síntomas y signos relacionados con neuropatía, enfermedad vascular periférica, y prevalencia de los mismos, y conocer el riesgo.

Materiales y métodos: se analizaron 165 pacientes en cuatro centros: Sanatorio Güemes (Servicio de Diabetes y Endocrinología), PREDIGMA (Centro de Medicina Preventiva, Posadas, Misiones), Hospital Central de San Isidro, Nexo Centro Médico (Ciudad de Junín) y Hospital Municipal de General Viamonte (Provincia de Buenos Aires).

Resultados: se encontró que el $43,6 \%$ presentaba algún síntoma en miembros inferiores y hasta el $57 \%$ alteración en las pruebas de tamizaje de neuropatía diabética o enfermedad arterial periférica, con mayor prevalencia a mayor tiempo de evolución de la DM. Los signos más frecuentemente hallados en el examen físico fueron: piel seca $(71,5 \%)$, distrofia ungueal $(60,6 \%)$ o alteración de la almohadilla plantar $(52,1 \%)$. Las comorbilidades más frecuentes fueron: hipertensión (74,5\%) y dislipemia $(73,3 \%)$. La mediana de hemoglobina glicosilada fue de $7,40 \%(6,70-$ $8,10)$, mayor en personas con antecedentes de retinopatía $(7,8 \% ; p<0,01)$ y en pacientes que refirieron tener calambres $(7,85$ a 7,$30 ; p=0,03)$ o ardor $(8,0$ vs 7,$3 ; p<0,01)$.

El porcentaje de pacientes con pie de alto riesgo por antecedentes, inspección o tamizaje de neuropatía o enfermedad vascular representó desde el $40 \%$ en aquellos con DM de menos de cinco años de evolución hasta el $86 \%$ en quienes tenían más de 20 años. Conclusiones: el elevado porcentaje de pacientes con pie de riesgo identificado en este estudio sugiere que, además del correcto examen físico, se requiere la toma de conductas por parte del médico tratante, como la indicación de plantillas o calzado adecuado, así como una fluida derivación al técnico en ortesis, traumatólogo o fisiatra.

Palabras clave: pie diabético; neuropatía diabética; tamizaje; diabetes mellitus; complicaciones.

Revista de la Sociedad Argentina de Diabetes 2021; Vol. 55 (04-12)

\section{ABSTRACT}

Introduction: foot examination is essential in patients with diabetes mellitus (DM). The correct evaluation of the foot in the patient who attends diabetes control is key to establish risk factors for the development of ulcers, detect injuries, take preventive measures, make an early referral and educate in foot care.

Objectives: in this study, the feet of people with DM were examined during the Diabetic Foot Awareness and Prevention Campaign on November 1, 2019 in order to assess the presence of symptoms and signs related to neuropathy, peripheral vascular disease, prevalence of the same and know the risk.

Materials and methods: 165 patients were analyzed in four centers: Sanatorio Güemes (Diabetes and Endocrinology Service), PREDIGMA (Preventive Medicine Center, Posadas, Misiones), Central Hospital of San Isidro, Nexo Medical Center (Junín City) and Hospital Municipal of General Viamonte (Province of Buenos Aires).

Results: it was found that $43.6 \%$ had some symptoms in the lower limbs and up to $57 \%$ had an alteration in the screening tests for diabetic neuropathy or peripheral arterial disease, with a higher prevalence the longer the evolution of DM.

The most frequent signs found in the physical examination were: dry skin $(71.5 \%)$, nail dystrophy (60.6\%) or alteration of the foot pad $(52.1 \%)$. The most frequent comorbidities were: hypertension (74.5\%) and dyslipidemia (73.3\%). The median glycated hemoglobin was $7.40 \%$ (6.70-8.10), higher in people with a history of retinopathy $(7.8 \% ; p<0.01)$ and in patients who reported having cramps (7,85 to 7.30; $p=0.03$ ) or burning (8.0 vs 7.3; $p<0.01)$. The percentage of patients with high-risk foot due to antecedents, inspection or screening for neuropathy or vascular disease represented from $40 \%$ in those with DM of less than five years of evolution to $86 \%$ in those who were older than 20 years.

Conclusions: this high percentage of patients with foot at risk identified in this study suggests that, in addition to the correct physical examination, the attending physician requires the taking of behaviors, such as the indication of appropriate footwear or insoles, as well as a fluid referral to the orthotic technician, orthopedic surgeon, or physiatrist.

Key words: diabetic foot; diabetic neuropathy; screening; diabetes mellitus; complications.

Revista de la Sociedad Argentina de Diabetes 2021; Vol. 55 (04-12) 
1 Comité de Pie Diabético de la Sociedad Argentina de Diabetes, Ciudad Autónoma de Buenos Aires, Argentina

2 Médica, especialista en Medicina Interna, Nutrición, Mágister en Diabetes, Diplomada en cicatrización de heridas, Coordinadora de Diabetes y Consultorio de Pie diabético, Servicio de Endocrinología y Diabetes, Sanatorio Güemes, Ciudad Autónoma de Buenos Aires, Argentina

3 Médica, especialista en Medicina Interna, Mágister en Diabetes, Unidad de Pie Diabético, Hospital Nacional Profesor Alejandro Posadas, Provincia de Buenos Aires, Argentina. Delegada International Working Group on the Diabetic Foot (IWGDF) por Argentina

4 Médico, especialista en Nutrición, Mágister en Diabetes, Hospital Municipal de Trauma y Emergencias Dr. Federico Abete, Pablo Nogués, Provincia de Buenos Aires, Centro de Diabetes y Enfermedades Metabólicas Dr. A. Maggio, Los Polvorines, Provincia de Buenos Aires, Argentina

5 Médica, especialista en Medicina Interna y Diabetes (Sociedad Argentina de Diabetes), Hospital Escuela de Agudos Dr. Ramón Madariaga/Centro de Medicina Preventiva (HEADRM/PREDIGMA), Posadas, Misiones, Argentina

6 Médico, especialista en Medicina General e Integral, Mágister en Diabetes, Servicio de Diabetología, Clínica Centro Junín, Hospital Municipal Gral. Viamonte, Consultorio de Pie Diabético HIGA Junín, Provincia de Buenos Aires, Argentina

7 Médico, especialista en Endocrinología, Hospital San Roque, Centro Integral de Atención al Diabético, San Salvador de Jujuy, Jujuy, Argentina

8 Médica, especialista en Nutrición, Universidad de Buenos Aires (UBA), Hospital Central de San Isidro, Provincia de Buenos Aires, Argentina

9 Médico, especialista en Medicina Interna, Nutrición y Diabetes, Hospital Alfredo I. Perrupato, San Martín, Mendoza, Argentina

10 Médica, especialista en Nutrición, habilitación especial Diabetología (Consejo Superior de la Pampa), Consultorio de Úlceras y Pie Diabético, Establecimiento Asistencial Gob. Centeno, La Pampa, Argentina

11 Médico Generalista y de Familia, Mágister en Diabetes, Diplomado en cicatrización de heridas, Servicio de Diabetes y Metabolismo, Hospital Regional de Río Grande, Tierra del Fuego, Argentina

12 Médica, especialista en Nutrición y Diabetes, Mágister en Nutrición Humana, Hospital Julio C. Perrando, Resistencia, Chaco, Argentina

13 Médica, especialista en Medicina Interna, Clínica de Imágenes, Centro de Endocrinología y Ría (CER), Neuquén, Argentina
14 Médica, especialista en Endocrinología, Hospital Centro de Salud Zenón Santillán, San Miguel de Tucumán, Tucumán, Argentina

15 Médico, Cirujano Cardiovascular, Hospital San Martín, Paraná, Entre Ríos, Argentina

16 Médica, especialista en Medicina Interna, Diplomada en Diabetología (Universidad de Ciencias Empresariales y Sociales, UCES), Hospital Sommer, Provincia de Buenos Aires, Argentina

17 Médica, especialista en Clínica Médica y Diabetes, Servicio de Endocrinología Nutrición y Diabetes, Hospital J. R. Vidal, Corrientes, Argentina

18 Médica, especialista en Medicina Interna, experta en Diabetes, Servicio de Diabetología y Nutrición, Hospital Privado Universitario de Córdoba, Córdoba, Argentina

19 Médica, especialista en Clínica Médica, Hospital Provincial de Rosario, Hospital Privado de Rosario, Santa Fe, Argentina

20 Médico, especialista en Endocrinología, Director del Programa de Pie Diabético, Hospital de Clínicas, Ciudad Autónoma de Buenos Aires, Argentina. Vice Chair de la International Working Group on the Diabetic Foot (IWGDF).

21 Médica, especialista en Medicina Interna, Mágister en Diabetes, Diplomada en cicatrización de heridas, Directora del Centro Municipal de Diabetes Dr. Alberto Maggio y del Hospital de Día de Pie Diabético Malvinas Argentinas, Provincia de Buenos Aires, Coordinadora del Comité de Pie Diabético de la Sociedad Argentina de Diabetes, Ciudad Autónoma de Buenos Aires, Argentina

22 Médica, especialista en Medicina Interna y Diabetes (Sociedad Argentina de Diabetes), Servicio de Endocrinología y Diabetes, Sanatorio Güemes, Ciudad Autónoma de Buenos Aires, Argentina

23 Médica, especialista en Medicina Interna y Endocrinología, Servicio de Endocrinología y Diabetes, Sanatorio Güemes, Ciudad Autónoma de Buenos Aires, Argentina

24 Médico, especialista en Medicina Interna y Endocrinología, Jefe del Servicio de Endocrinología y Diabetes, Sanatorio Güemes, Ciudad Autónoma de Buenos Aires, Argentina

Contacto de la autora: Claudia Marcela Issa

E-mail: clauissa@gmail.com

Correspondencia: Fraga $17401^{\circ} \mathrm{B}$ (C1427BUJ), Ciudad Autónoma de Buenos Aires, Argentina Autónoma de Buenos

Aires, Argentina

Fecha de trabajo recibido: 29/10/20

Fecha de trabajo aceptado: 23/12/20

Conflictos de interés: los autores declaran que no existe conflicto de interés.

\section{INTRODUCCIÓN}

Entre las complicaciones crónicas de la diabetes mellitus (DM) se encuentra la neuropatía diabética (ND) y una de sus consecuencias más graves es el pie diabético (PD).

EI PD se define como la ulceración, infección y/o gangrena del pie asociados a ND y diferentes grados de enfermedad arterial periférica (EAP) ${ }^{1}$.

Aproximadamente del 15 al $35 \%$ de los pacientes con DM tendrán, en el transcurso de la enfermedad, úlceras en las extremidades inferiores, de las cuales del 7 al 20\% requerirán posteriormente amputación².

La ND es el factor fundamental en la fisiopatología del PD, y se estima que su prevalencia oscila entre el 6 y el $51 \%$ de los adultos con DM según de la duración de la enfermedad, la edad y el control glucémico ${ }^{3}$. Algunas de las alteraciones involucradas en la etiopatogenia de la ND son el incremento de la vía de los polioles, la glicación de las proteínas, la acción de la proteína quinasa $\mathrm{C}$ y el estrés oxida- 
tivo, entre otras ${ }^{4}$.La misma puede cursar en forma asintomática hasta en el $50 \%$ de los pacientes que la padece. Su falta de reconocimiento no permite implementar medidas preventivas en forma oportuna y hace que los pacientes se encuentren en riesgo de desarrollar úlceras e infecciones en el pie, lo cual incrementa el riesgo de amputación ${ }^{5}$.

La EAP puede detectarse hasta en el $50 \%$ de los pacientes con úlceras en pie diabético 6 . La presencia de DM confiere un estado metabólico anormal que favorece la aterosclerosis acelerada de todo el árbol vascular, con cambios proinflamatorios, reológicos y hemostáticos que modifican el equilibrio entre la trombosis y la fibrinolisis?. La presencia de EAP predice mal pronóstico en cuanto a la cicatrización de las úlceras ${ }^{5,8}$, y también aumenta de forma independiente el riesgo de amputación mayor y mortalidad en un paciente con $\mathrm{PD}^{6}$. Si bien muchos pacientes son asintomáticos hasta que aparecen las lesiones en el pie ${ }^{6}$, la EAP puede detectarse mediante el interrogatorio (antecedentes de claudicación o dolor en reposo) o durante el examen físico (palpación de pulsos y características tróficas) y luego confirmarse con estudios complementarios.

La anamnesis y el examen rutinario del pie en un paciente con DM permiten detectar factores que aumentan el riesgo de amputación, como los antecedentes de úlceras y amputaciones previas, la insuficiencia renal crónica en diálisis, el tabaquismo (debe registrarse el consumo de tabaco dado que es un factor de riesgo no sólo de enfermedad vascular sino también de neuropatía), las deformidades y lesiones pre ulcerativas causadas por la neuropatía motora, la pérdida de sensibilidad protectora secundaria a la neuropatía sensitiva y el estado circulatorio ${ }^{5,9}$. De aquí se desprende la importancia de la correcta evaluación del pie en un paciente que consulta para el control de su DM porque constituye un elemento que le permite al médico tratante evaluar el riesgo que ese paciente tiene de presentar una lesión y tomar una conducta acertada.

Durante 2019 se desarrolló -a partir del Comité de Pie Diabético de la Sociedad Argentina de Diabetesla primera Campaña Latinoamericana relacionada con actividades de prevención de pie diabético, y se eligió el $1^{\circ}$ de noviembre como el Día Latinoamericano de la Concientización y Prevención del Pie Diabético. En el marco de dicha campaña, se llevó a cabo la difusión de videos de prevención, salas de espera interactivas, charlas a la comunidad y actividades en conjunto con pacientes y otros profesionales.

\section{OBJETIVOS}

En este contexto, se desarrolló un protocolo en cuatro instituciones que consistió en el examen de pies de personas con DM que concurrieron en forma voluntaria a los centros con el objetivo de determinar qué porcentaje que asistió a la consulta durante la campaña presentó factores de riesgo para el desarrollo de una úlcera en pie y/o síntomas y signos relacionados con neuropatía y enfermedad vascular periférica.

\section{MATERIALES Y MÉTODOS}

Estudio multicéntrico, descriptivo, observacional de corte transversal. La recolección de datos se realizó en el Sanatorio Güemes (Servicio de Diabetes y Endocrinología), en PREDIGMA (Centro de Medicina Preventiva, Posadas, Misiones), en el Hospital Central de San Isidro, en Nexo Centro Médico (Ciudad de Junín) y en el Hospital Municipal de General Viamonte (Provincia de Buenos Aires).

Los datos se registraron en una planilla unificada, formulario electrónico de registro de casos el cual se diseñó específicamente para dicho fin; se preservó el anonimato de los participantes que firmaron un consentimiento informado para ingresar en nuestra institución de acuerdo a las normativas de la misma al igual que en el resto de los centros participantes.

Se incluyeron pacientes mayores de 18 años con diagnóstico de DM, que concurrieron espontáneamente para realizar el examen de pies en el contexto de la Campaña Latinoamericana de Concientización y Prevención del Pie Diabético el $1^{\circ}$ de noviembre de 2019. Se recolectaron datos filiatorios, antecedentes de amputación mayor (infrapatelar o supracondílea), menor (transmetatarsiana, rayos, dedos) o úlceras en pie, comorbilidades (insuficiencia cardíaca, enfermedad coronaria, dislipidemia, hipertensión arterial, nefropatía, insuficiencia renal crónica -IRC- en diálisis, tabaquismo), y se interrogó acerca de la presencia de síntomas de neuropatía (dolor, ardor, parestesias, calambres) y claudicación. Se realizó examen de pies mediante monofilamento, diapasón, sensibilidad térmica y reflejos osteotendinosos, y se evaluó la presencia de deformidades, alteraciones o lesiones pre ulcerativas (dedos en garra, en martillo, pérdida de almohadilla plantar, ausencia de pulsos, falta de vello, engrosamiento de las uñas). Se realizó registro de tensión arterial sistólica en ambos brazos y tobillos utilizando doppler portátil bidireccional con transductor de $8 \mathrm{Mhz}$. Los pacientes fueron pesados y 
medidos, y se calculó el índice de masa corporal (IMC). También se registraron datos de hemoglobina glicosilada ( $\mathrm{HbA} 1 \mathrm{c})$, colesterol LDL y creatinina.

Los investigadores, quienes tenemos experiencia en investigación, adherimos plenamente a la Ley Nacional de Protección de Datos Personales ( $N^{\circ}$ 25.326), la Declaración de Helsinki de la Asociación Médica Mundial y las pautas éticas internacionales para las investigaciones biomédicas en seres humanos del Consejo de Organizaciones Internacionales de las Ciencias Médicas -CIOMS-6. Basados en estos conceptos, los únicos que tuvieron acceso a los datos identificatorios de los participantes y procedieron al enmascaramiento de la información fueron los dos autores principales y, de esta manera, se aseguró que no sea posible identificar la inclusión de ningún individuo en el presente estudio.

\section{Análisis estadístico}

Las variables categóricas se presentaron como valor absoluto y porcentaje. Las variables continuas como media y desvío standard cuando su distribución fue simétrica, o mediana e intervalo intercuartil cuando su distribución fue asimétrica. Se realizó análisis de variables categóricas con test de chi cuadrado y de variables continuas con test de student en caso de distribución simétrica o Mann Whitney en casos de distribución asimétrica. Para el cálculo de la varianza no paramétrica se utilizó la prueba de Kruskal Wallis. Para el análisis de los datos se utilizaron los programas de Infostat y Vcc stat.

\section{RESULTADOS}

Se analizaron 165 pacientes que concurrieron a cuatro centros. De ellos, 83 (50,3\%) fueron de sexo femenino. La mediana de edad fue de 61 años (53-70). De los pacientes analizados, 30 (18\%) tenían menos de cinco años de evolución de la DM, 53 (32\%) entre 5 y 10 años de evolución, 53 (32\%) de 10 a 20 años de evolución y 28 (17\%) más de 20 años de evolución. En un paciente se desconoció el tiempo de evolución. En la Tabla 1 se describe la frecuencia de las comorbilidades asociadas a la DM y fue la hipertensión arterial la más frecuente $(74,5 \%)$, seguida por dislipemia (73,3\%). Sólo 28 pacientes (16,9\%) refirieron no tener comorbilidad. Al interrogatorio, el $41 \%$ de los pacientes $(n=67)$ refirió algún tipo de ingesta de alcohol y el $23 \%(n=38)$ tabaquismo. Los antecedentes mencionados por los pacientes se describen en la Tabla 2 y el más frecuente fue la úlcera en pie en el $15,8 \%$ de los casos $(n=26)$. La media de peso fue de $82,97 \mathrm{~kg}(18,1)$, con una media de $75,24 \mathrm{~kg}$ para las mujeres y de 90,71 kg para los hombres $(p<0,01)$. El promedio de IMC fue de $30,26(6,17), 29,95$ para las mujeres y 30,58 para los hombres $(p=0,51)$. El IMC fue mayor en pacientes hipertensos $(30,89$ vs 28,$45 ; p=0,03)$ y en aquellos con antecedentes de accidente cerebrovascular (ACV) $(38,17$ vs 30,01; $p=0,003)$.

Los pacientes con antecedentes de amputación menor $(n=10)$ tenían una mediana de 15 años de evolución de la DM contra ocho años de aquellos que no tenían dicho antecedente $(p<0,01)$. Los pacientes con antecedentes de amputación mayor $(n=2)$ tenían una mediana de 34 años de evolución de la DM $(p=0,02)$.

La mediana de presión en el tobillo fue de $130 \mathrm{mmHg}$. Dicho valor no tuvo diferencias significativas según los años de evolución de la DM.

En relación a los síntomas de miembros inferiores, 72 pacientes $(43,6 \%)$ refirieron presentar al menos uno. Los síntomas más frecuentes hallados al interrogatorio directo fueron los calambres en el $32 \%$, llegando al $57 \%$ de los pacientes con DM de más de 20 años de evolución, seguido por hormigueos $(30 \%)$, ardor $(19 \%)$, dolor (9\%) y claudicación $(8 \%)$. Salvo en el caso del dolor, el resto de los síntomas aumentó con el tiempo de evolución de la DM en forma estadísticamente significativa $(p<0,05)$ (Gráfico 1).

Los signos de ND o alteraciones vinculadas a EAP encontrados en el examen de pie se describen en la Tabla 3. La piel seca, la distrofia ungueal y la alteración en la almohadilla plantar fueron los signos más frecuentemente detectados.

La mediana de $\mathrm{HbA} 1 \mathrm{c}$ en los pacientes evaluados fue de $7,40(6,70-8,10)$. La misma se registró en 147 pacientes y su valor fue variable según el tiempo de evolución de la DM (Gráfico 2). La mediana de $\mathrm{HbA} 1 \mathrm{c}$ fue mayor $(7,85$ a 7,30$)$ en pacientes que refirieron tener calambres $(p=0,03)$ y también en quienes manifestaron sentir ardor $(8,0$ vs 7,3$)(p<0,01)$. No hubo diferencias en la HbA1c con respecto a otros síntomas.

Tanto la retinopatía como la nefropatía, enfermedad coronaria, hipertensión arterial, ACV y dislipemia aumentaron su frecuencia en relación a los años de evolución de la DM (Gráfico 3). Los pacientes que refirieron tener diagnóstico de retinopatía presentaron una mediana de $\mathrm{HbA} 1 \mathrm{c}$ mayor $(7,8 \%)$ que aquellos que no refirieron ese diagnóstico $(7,3 \% ; p<0,01)$. En el caso de la nefropatía, los valores fueron 7,65 y $7,4 \%$ respectivamente $(p=0,09)$, y en el caso de 
antecedentes de úlcera en pie 7,8 y $7,3 \%$ respectivamente $(p=0,07)$. Para otras comorbilidades (ACV, enfermedad coronaria, insuficiencia cardíaca) no hubo diferencias en la mediana de $\mathrm{HbA} 1 \mathrm{c}$.

Se realizó prueba de diapasón y se halló que en 94 pacientes (57\%) estaba alterada. También se encontraron alteraciones en la prueba de monofilamento en 66 pacientes (40\%), en la de sensibilidad térmica en $71(43 \%)$ y en reflejos osteotendinosos en $87(53 \%)$. El 54,5\% de los pacientes $(n=90)$ presentó alterada más de una prueba. La mediana de edad fue mayor (67 años) para quienes tuvieron prueba de monofilamento alterada que para aquellos con prueba normal (58 años; $p<0,01$ ). En el caso de la prueba de diapasón y de los reflejos osteotendinosos, 67 años vs 56 años $(p<0,01)$, y para la sensibilidad térmica 66 a 58 años $(p<0,01)$. Como se muestra en el Gráfico 4, la presencia de alteración en las pruebas de detección de ND aumentó a mayor tiempo de evolución de la DM.

En relación a las conductas de prevención, 108 pacientes $(65 \%)$ manifestaron realizar autocuidados, 103 (62\%) utilizaban medias adecuadas al momento del examen y 106 (64\%) calzado adecuado. Solamente 10 pacientes (6\% de la muestra) no presentaron alteración que aumentara el riesgo de neuropatía relacionada al interrogatorio, inspección, síntomas y pruebas de tamizaje de ND o EAP.

\begin{tabular}{|l|r|r|}
\hline Comorbilidades & $\mathbf{n ~ ( \% )}$ & IC $\mathbf{9 5} \%$ \\
\hline Hipertensión arterial & $123(74,5)$ & $67-80,9$ \\
\hline Dislipemia & $121(73,3)$ & $65,8-79,8$ \\
\hline Retinopatía & $47(28,5)$ & $22,4-36,8$ \\
\hline Enfermedad coronaria & $27(16,4)$ & $11,2-23,1$ \\
\hline Nefropatía & $27(16,4)$ & $11,2-23,1$ \\
\hline Insuficiencia cardíaca & $11(6,7)$ & $3,5-11,9$ \\
\hline Pie de Charcot & $3(1,8)$ & $0,4-6,7$ \\
\hline IRC diálisis & $2(1,2)$ & $0,2-4,5$ \\
\hline
\end{tabular}

IRC: insuficiencia renal crónica

Tabla 1: Comorbilidades asociadas a la diabetes.

\begin{tabular}{|l|r|r|}
\hline Antecedentes & $\mathbf{n ~ ( \% )}$ & IC 95\% \\
\hline Antecedentes de úlcera en pie & $26(15,8)$ & $10,7-22,4$ \\
\hline Antecedentes de amputación menor & $10(6,1)$ & $3,1-11,2$ \\
\hline Antecedentes de accidente cerebrovascular & $5(3)$ & $0,9-11,1$ \\
\hline Antecedentes de angioplastia & $4(2,4)$ & $0,7-7,1$ \\
\hline Antecedentes de amputación mayor & $2(1,2)$ & $0,2-4,5$ \\
\hline Insuficiencia cardíaca & $11(6,7)$ & $3,5-11,9$ \\
\hline Pie de Charcot & $3(1,8)$ & $0,4-6,7$ \\
\hline IRC diálisis & $2(1,2)$ & $0,2-4,5$ \\
\hline
\end{tabular}

IRC: insuficiencia renal crónica

Tabla 2: Antecedentes en los pacientes con diabetes.

\begin{tabular}{|l|r|r|}
\hline Tipo de alteración & $\mathbf{n}(\mathbf{\%})$ & IC 95\% \\
\hline Piel seca & $118(71,5)$ & $63,9-78,1$ \\
\hline Onicodistrofia & $100(60,6)$ & $52,7-60$ \\
\hline Alteración en la almohadilla plantar & $86(52,1)$ & $44,2-59,9$ \\
\hline Hiperqueratosis & $83(50,3)$ & $42,5-58,1$ \\
\hline Micosis/intertrigo & $67(40,6)$ & $33,1-48,5$ \\
\hline Dedos en garra & $68(41,2)$ & $33,7-49,1$ \\
\hline Venas dilatadas & $60(36,4)$ & $29,1-44,2$ \\
\hline Ausencia de vello & $50(30,3)$ & $23,4-38,0$ \\
\hline Alteración en el apoyo del metatarso & $44(26,7)$ & $20,2-34,2$ \\
\hline Ausencia de pulsos & $35(21,2)$ & $15,2-28,6$ \\
\hline Relleno capilar alterado & $34(20,6)$ & $14,7-27,6$ \\
\hline Grietas & $14(8,5)$ & $4,9-14,1$ \\
\hline Dedos en martillo & $11(6,7)$ & $3,5-11,9$ \\
\hline Dedos en masa & $9(5,5)$ & $2-15,3$ \\
\hline Maceración & $6(3,6)$ & $1,2-12,4$ \\
\hline
\end{tabular}

Tabla 3: Presencia de alteraciones al examen físico.

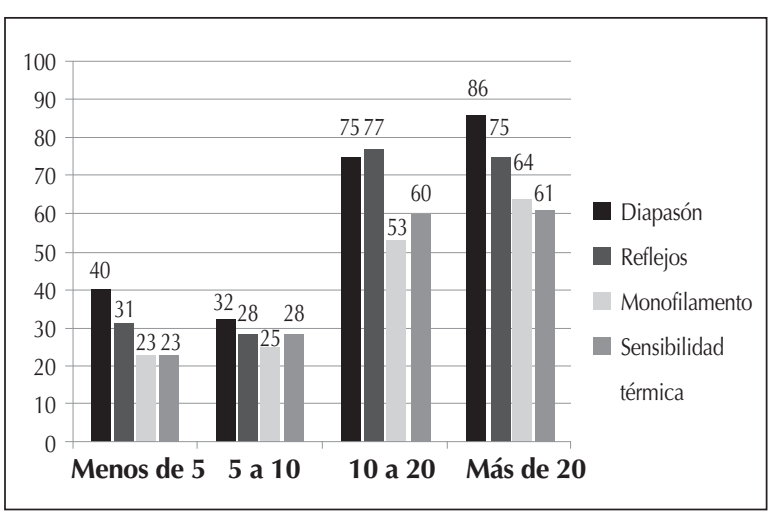

(*) p menor a 0,05

Gráfico 1: Síntomas de miembros inferiores en relación a la duración de la diabetes.

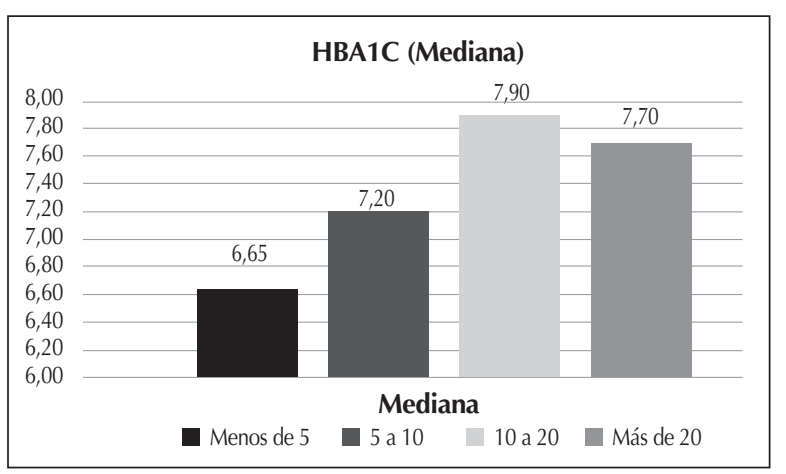

Gráfico 2: Mediana de HbA1c según tiempo de evolución de la diabetes $(n=147)$. 


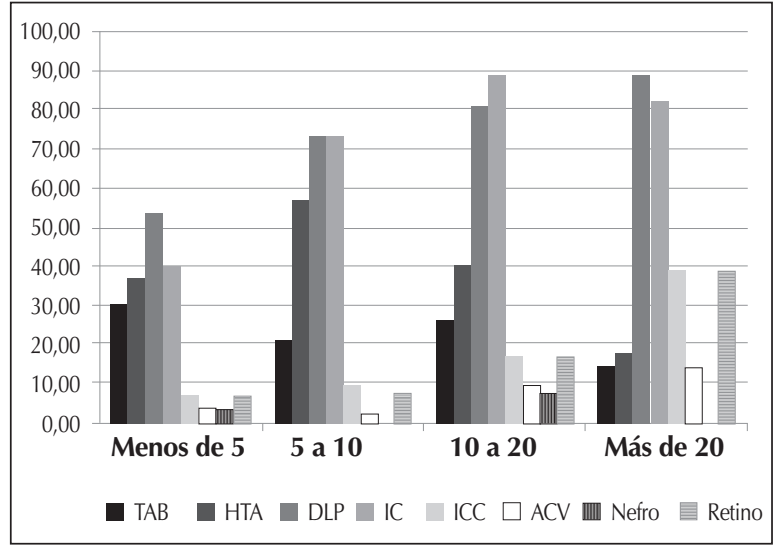

TAB: tabaco; HTA: hipertensión arterial; DLP: dislipemia; IC: isquemia coronaria ; ICC: insuficiencia cardíaca congestiva; ACV: accidente cerebrovascular; Nefro: nefropatía; Retino: retinopatía. Todos $P$ menor 0,05 excepto ACV

Gráfico 3: Prevalencia de comorbilidades en relación a la duración en años de la diabetes.

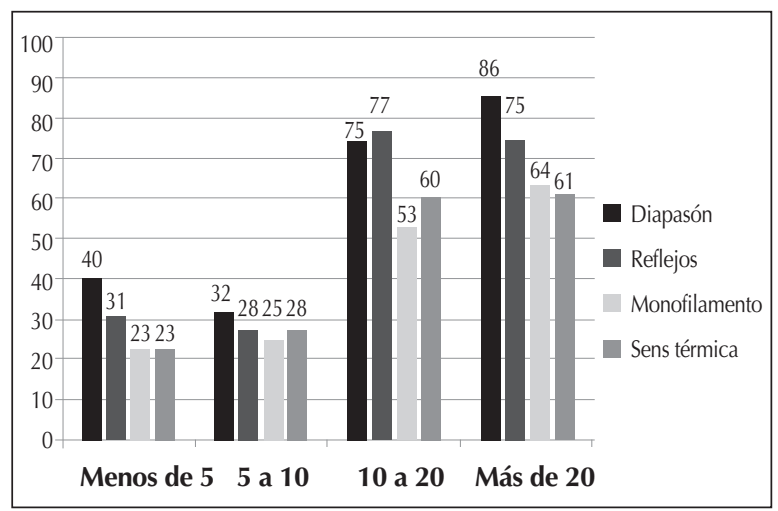

p $<0,05$ para todas las pruebas. Sens térmica $=$ prueba de sensibilidad térmica (fibras finas).

Gráfico 4: Proporción de pacientes con alteración en las pruebas de neuropatía según los años de evolución de la diabetes.

\section{DISCUSIÓN}

Las recomendaciones acerca del examen periódico y oportuno del pie, las pruebas de tamizaje para detectar ND y EAP, así como la educación como elementos de prevención de lesiones de pie en pacientes con DM tienen una recomendación fuerte por parte de todas las guías pero una evidencia baja ${ }^{10,11}$. Sin embargo, el $85 \%$ de las amputaciones de miembros inferiores en pacientes con DM es precedida por una úlcera, con lo cual los esfuerzos deben focalizarse en su prevención.

Los factores descriptos en la bibliografía asociados a un aumento del riesgo para futuras ulceraciones de pie en pacientes con DM incluyen antecedentes de amputación de miembros inferiores (aumentan 2,8 veces el riesgo), antecedentes de úlcera previa (1,6 veces), duración de la DM de más de 10 años (tres veces), HbA1c mayor a $9(3,2 \text { veces })^{9}$. En nuestro estudio, el $12 \%$ de los pacientes tenía antecedentes de amputación, el $26 \%$ de úlcera previa y el $49 \%$ más de 10 años de evolución de su DM.

En relación a las pruebas de tamizaje de ND, la prueba de monofilamento tiene una sensibilidad y especificidad variables para la detección de pérdida de sensibilidad protectora que va del 66 al $91 \%$ en el primer caso y del 34 al $86 \%$ en el segundo. En nuestro estudio encontramos que la alteración en la prueba de monofilamento se incrementó con la duración de la DM y con la edad del paciente, y se halló que aquellos con más de 20 años de evolución de su DM tuvieron pérdida de la sensibilidad protectora hasta en el $64 \%$ de los casos. En un estudio reciente ${ }^{12}$, en el cual se enrolaron 323 pacientes con DM2, la presencia de polineuropatía se asoció a mayor duración de la DM y al aumento del valor de la hemoglobina glicosilada. En nuestro estudio, la presencia de síntomas relacionados con neuropatía (ardor, calambres y hormigueo) y la alteración de las pruebas de detección de neuropatía (monofilamento, diapasón, reflejos y sensibilidad térmica) también se asociaron a mayor duración de la DM. La presencia de ardor y calambres se relacionaron, además, con valores mayores de HbA1c. Esto también se menciona en varios estudios donde se describe que un aumento en la $\mathrm{HbA} 1 \mathrm{c}$ del $2 \%$ conduce a un incremento del riesgo de padecer neuropatía de 1,6 veces en un período de cuatro años ${ }^{13,14}$.

La prevalencia de alteraciones en el examen físico de los pies hallada en este estudio (que llega hasta más del $70 \%$ en algunos casos) se correlacionó con los datos encontrados en un estudio realizado en 11.357 pacientes internados en hospitales de Latinoamérica, donde se detectó que, de igual manera, sólo el $21 \%$ de los pacientes internados con DM no tenía alteración en el pie, mientras que el resto presentaba úlceras o lesiones pre ulcerativas ${ }^{15}$.

Las guías sugieren no realizar intervenciones específicas en pacientes sin factores de riesgo (pérdida de sensibilidad protectora, deformidad, EAP o antecedentes de úlcera o amputación), pero es muy importante destacar que en nuestro estudio solamente 10 pacientes de los 165 estu- 
diados no tuvieron alteración que pudiera conferir riesgo de amputación como deformidades (dedos en garra, en martillo, alteración del apoyo del metatarso), lesiones pre ulcerativas (hiperqueratosis, grietas, micosis) o alteraciones en las pruebas de detección de ND (diapasón, temperatura, monofilamento, reflejos); el resto presentaba al menos una. Además, el porcentaje de pacientes con alteración de alguno de dichos aspectos mencionados fue desde un $40 \%$ en aquellos con DM de menos de cinco años de evolución hasta un $86 \%$ en quienes tenían más de 20 años. Por lo tanto, y al no haber evidencia concreta sobre prevención primaria, las recomendaciones deben adaptarse contemplando las características de la población de pacientes que consulta que, además, se relacionan con la edad y el nivel socioeconómico y cultural, entre otros factores.

La importancia de este estudio radica en el conocimiento de que la población local presenta un alto riesgo de desarrollar úlceras, y las acciones deben adaptarse a dicha realidad al reforzar, además, la estrategia de tamizaje con pruebas de NP, EAP e inspección, la toma de decisiones y derivación precoz para adecuar plantillas y calzado ante los hallazgos del examen físico e interrogatorio. Examinar los pies de los pacientes con DM en cada consulta por el médico tratante es fundamental para iniciar una estrategia de prevención y manejo de las úlceras en pie diabético, pero a menudo es insuficiente. Teniendo en cuenta los resultados de este estudio, los pacientes mayores y con mayor duración de la DM son los que presentan mayor riesgo de desarrollar úlceras en pie. Por lo tanto, la mayoría requiere una intervención adicional, además de la observación, y sería adecuado que el médico que los atiende se comunique fluidamente con un podólogo, traumatólogo especializado en pie, un médico fisiatra o un equipo de pie diabético a fin de realizar la indicación de la descarga correspondiente a la brevedad según corresponda.

El examen clínico debe acompañarse de la indicación de calzado adecuado (zapatillas deportivas) y plantillas si existen zonas de hiperqueratosis o deformidades, además del refuerzo de las medidas de prevención (como evitar caminar descalzo o en ojotas, corte de uñas, etc.). Si no se dispone de tiempo durante la consulta, evaluar los pies mediante el examen de los 3 minutos $^{16}$ (Cuadro 1) permitirá identificar, en un lapso corto de tiempo, lesiones pre ulcerativas y factores de riesgo, y de- rivar a la brevedad al equipo de pie diabético para realizar las indicaciones correspondientes. Deben estudiarse cuáles son las causas más frecuentes de lesiones en los pies, el nivel de educación e ideas previas que presentan los pacientes, el nivel de conocimiento de los médicos de atención primaria y especialistas en DM para dirigir las acciones de prevención.

En cuanto a los pacientes, la educación debe estructurarse con estrategias grupales, individuales, motivacionales, videos, folletos, talleres de prevención; todas estas estrategias deben ser culturalmente apropiadas y adaptadas. Algunos estudios sugieren que la educación del paciente acerca del pie diabético y su prevención mejora el conocimiento a largo plazo y puede reducir en forma modesta las ulceraciones y las amputaciones $^{9}$, sin embargo no hay evidencia sólida de que la educación limitada hacia el paciente por sí sola sea eficaz para lograr reducciones clínicamente relevantes de la incidencia de úlceras y amputaciones $^{17}$. La difusión del problema del PD debe ser masiva, hacia la población general, que haga conocer su existencia y comprometer a los pacientes en su propio cuidado. Deben utilizarse medios de comunicación, redes sociales, telesalud y todos los elementos disponibles para alertar tanto a la población general como así también a médicos y funcionarios que se trata de un verdadero problema de salud pública. En cuanto a los profesionales y el sistema de salud, es necesario fortalecer las redes de derivación y la incorporación de podólogos y ortesistas en los distintos niveles de atención. En este contexto podría incorporarse algún sistema de historia clínica unificada o cartilla de seguimiento para mejorar la comunicación entre profesionales a fin de empoderar al paciente acerca de la importancia del tratamiento, así como la implementación de un consultorio de prevención de pie diabético con sistema de derivación rápida desde el médico clínico o diabetólogo.

Tal como se muestra en el cuadro de riesgo adaptado por el Comité de Pie Diabético de la Sociedad Argentina de Diabetes (Cuadro 2), la presencia de deformidad del pie (dedos en garra, hallux valgus, dedos en martillo) evaluada en el examen físico ya confiere al pie un riesgo moderado que amerita la indicación de calzado adecuado y la valoración de confección de plantillas. Más aún, si aparecen hiperqueratosis $u$ otras lesiones pre ulcerativas, también evaluables en el examen físico, la 
indicación de calzado adecuado y plantillas debe ser prioritaria ya que el riesgo es muy alto.

En vista de los resultados de este estudio, más de la mitad de los pacientes presentó alguna lesión pre ulcerativa independientemente de las pruebas realizadas con diapasón o monofilamento. Por lo tanto, el examen físico se convierte en una herramienta indispensable e insustituible dado que permite detectar la mayoría de las alteraciones que aumenta el riesgo de padecer una úlcera y actuar en consecuencia con una simple evaluación que no requiere técnicas complejas ni aparatología (inspección, test de Ipswich, palpación de pulsos) ${ }^{18}$. Consideramos como perspectivas futuras, y en relación a los resultados obtenidos, el diseño de un estudio prospectivo de análisis de la presión plantar y consecuente intervención con la confección de plantillas, calzado y educación estructurada en el grupo de mayor riesgo de ulceraciones, como aquellos pacientes con más de 20 años de evolución o mayores de 60 años, y evaluar el costo-beneficio para la prevención primaria de úlceras en pie diabético.

\begin{tabular}{|c|c|c|}
\hline Minuto 1 & Minuto 2 & Minuto 3 \\
\hline $\begin{array}{l}\text { Antecedentes del paciente } \\
\text { - ¿Tiene diabetes? ¿Desde cuándo? } \\
\text { ¿Cómo están sus monitoreos? } \\
\text { - ¿Presentó úlceras, amputaciones o } \\
\text { cirugía en miembros inferiores? } \\
\text { - ¿Se le realizó angioplastia, } \\
\text { colocación de stent o bypass en } \\
\text { miembros inferiores? } \\
\text { - ¿Presenta úlcera actualmente? } \\
\text { - ¿Fuma? ¿Consume alcohol? } \\
\text { Estado actual del paciente } \\
\text { - ¿Ardor u hormigueos en pies o piernas? } \\
\text { - ¿Dolor o fatiga al caminar? } \\
\text { - ¿Cambios en la coloración de la piel o } \\
\text { lesiones? } \\
\text { - ¿Pérdida de sensibilidad de miembros } \\
\text { inferiores? } \\
\text { ¿Consulta regularmente al podólogo para } \\
\text { mantener el cuidado de sus pies? }\end{array}$ & $\begin{array}{l}\text { Examen vascular } \\
\text { - Palpar pulso pedio y tibial posterior } \\
\text { - Evaluar temperatura de la piel } \\
\text { - ¿Presenta vello distal en los pies? } \\
\text { - Evaluar tiempo de relleno capilar en los dedos de los pies } \\
\text { Examen neurológico } \\
\text { - Evaluar pérdida de sensibilidad protectora utilizando test de } \\
\text { monofilamento de Semmes-Weinstein o diapasón o prueba } \\
\text { táctil de Ipswich } \\
\text { Examen músculo esquelético } \\
\text { - Buscar deformidades óseas } \\
\text { - Evaluar movilidad del tobillo y primera articulación } \\
\text { metatarsofalángica } \\
\text { - Buscar signos de pie de Charcot (pie caliente, rojo, hinchado, } \\
\text { arco colapsado) } \\
\text { Examen dermatológico } \\
\text { - Observar las uñas, evaluar la presencia de micosis ungueal } \\
\text { o interdigital } \\
\text { - Evaluar callosidades, grietas y maceración de la piel }\end{array}$ & $\begin{array}{l}\text { Referir } \\
\text { - Evaluar al paciente para determinar } \\
\text { el riesgo y valorar seguimiento } \\
\text { Educación del paciente } \\
\text { - Informarlo sobre el riesgo de } \\
\text { ulceración y amputación } \\
\text { - Recordarle mirarse los pies } \\
\text { diariamente } \\
\text { - Educarlo sobre los riesgos de } \\
\text { caminar descalzo, incluso en su casa } \\
\text { - Sugerirle el uso de calzado } \\
\text { adecuado } \\
\text { - Aconsejarle sobre el cese del hábito } \\
\text { tabáquico } \\
\text { - Recomendarle sobre el control } \\
\text { adecuado de su glucemia, presión } \\
\text { arterial y dislipemia } \\
\text { - Educarlo sobre cómo llevar un plan } \\
\text { de alimentación equilibrado y la } \\
\text { importancia de realizar actividad } \\
\text { física en forma regular }\end{array}$ \\
\hline
\end{tabular}

Cuadro 1: Cuadro de riesgo adaptado por el Comité de Pie Diabético de la Sociedad Argentina de Diabetes.

\begin{tabular}{|c|c|c|c|c|}
\hline Riesgo bajo & Riesgo moderado & Riesgo alto & Riesgo muy alto & Riesgo extremo \\
\hline $\begin{array}{l}\text { - Sensación protectora } \\
\text { plantar normal } \\
\text { - Pulsos pedio/tibial } \\
\text { posterior positivos. } \\
\text { Sin deformidad }\end{array}$ & $\begin{array}{l}\text { - PSP o EAP o deformidad } \\
\text { (un solo factor) }\end{array}$ & $\begin{array}{l}\text { - PSP+EAP } \\
\text { - PSP+deformidad } \\
\text { - EAP+deformidad } \\
\text { (dos o tres factores) } \\
\text { - Onicomicosis } \\
\text { - Lesiones pre ulcerativas }\end{array}$ & $\begin{array}{l}\text { - Úlcera previa } \\
\text { - Amputación previa } \\
\text { - Charcot } \\
\text { - ERCT }\end{array}$ & $\begin{array}{l}\text { - Ataque de pie diabético } \\
\text { (foot attack) } \\
\text { - Isquémico, infectado, } \\
\text { Charcot agudo, mixto }\end{array}$ \\
\hline \multicolumn{5}{|c|}{ Seguimiento acorde al riesgo } \\
\hline $\begin{array}{l}\text { Inspección en cada visita } \\
\text { Examen al año }\end{array}$ & $\begin{array}{l}\text { Inspección en cada visita } \\
\text { Examen a los 3-6 meses }\end{array}$ & $\begin{array}{l}\text { Inspección en cada visita } \\
\text { Examen a los } 3 \text { meses }\end{array}$ & $\begin{array}{l}\text { Inspección en cada visita } \\
\text { Examen en un mes }\end{array}$ & $\begin{array}{l}\text { Derivación inmediata: } \\
\text { guardia }\end{array}$ \\
\hline \multicolumn{5}{|c|}{ Educación del paciente y de su entorno. Controles por podología } \\
\hline \multicolumn{5}{|c|}{ Control metabólico adecuado } \\
\hline $\begin{array}{l}\text { Recomendación de calzado } \\
\text { apropiado }\end{array}$ & $\begin{array}{l}\text { - Calzado adecuado } \\
\text { indicado por profesional } \\
\text { capacitado } \\
\text { - Valorar tipo de plantilla }\end{array}$ & $\begin{array}{l}\text { - Calzado y plantillas } \\
\text { adecuados, indicados por } \\
\text { profesional capacitado } \\
\text { - Tratamiento de micosis y } \\
\text { lesiones preulcerativas }\end{array}$ & $\begin{array}{l}\text { - Calzado y plantillas con } \\
\text { modificaciones perso- } \\
\text { nalizadas, indicadas por } \\
\text { profesional capacitado } \\
\text { - Valorar descargas }\end{array}$ & $\begin{array}{l}\text { - Evaluar internación } \\
\text { - Valorar cirugía de } \\
\text { urgencia } \\
\text { - ATB de amplio espectro } \\
\text { - Avisar al equipo de pie } \\
\text { diabético }\end{array}$ \\
\hline
\end{tabular}

PSP: pérdida de sensibilidad protectora; EAP: enfermedad arterial periférica; ERCT: enfermedad renal crónica terminal.

Cuadro 2: Evaluación del riesgo en pie diabético. Adaptado por el Comité de Pie Diabético de la Sociedad Argentina de Diabetes según las guías de la Asociación Latinoamericana de Diabetes (ALAD), la International Diabetes Federation (IDF), la International Working Group on the Diabetic Foot (IWGDF) y la American Diabetes Association (ADA). 


\section{CONCLUSIONES}

Las comorbilidades más frecuentemente encontradas en pacientes con DM fueron hipertensión arterial y dislipemia. La prevalencia de comorbilidades aumentó junto con la duración de la DM.

La piel seca, la distrofia ungueal, la disminución de la almohadilla plantar y la hiperqueratosis fueron las alteraciones más frecuentes halladas en el examen físico en más de la mitad de los pacientes.

El $43,6 \%$ de los pacientes refirió tener al menos un síntoma relacionado a neuropatía o enfermedad arterial periférica (calambres, ardor, dolor, hormigueo, claudicación).

La mediana de HbA1c aumentó con la duración de la DM, fue mayor en pacientes con antecedentes de retinopatía y en aquellos que refirieron tener calambres o ardor.

Entre las pruebas de detección de ND, la alteración en la prueba de diapasón fue la más frecuente encontrada en más de la mitad de los pacientes evaluados. El porcentaje de pacientes con alteración en las pruebas de tamizaje de ND aumentó con la duración de la DM y la edad.

De todos los pacientes evaluados, menos del $10 \%$ no presentó prueba de tamizaje de ND o EAP alterada, ningún hallazgo en el examen físico, ningún síntoma de miembros inferiores, ni tenía antecedentes que aumentaran el riesgo de su pie (amputaciones o úlceras previas).

Más de la mitad de los pacientes evaluados usaba medias y calzado adecuados, y refirió conductas de autocuidado.

\section{Dedicatoria}

Dr. Javier Aníbal Vilte hasta siempre. Desde el Comité de Pie Diabético queremos resaltar tu trayectoria como Miembro Titular de la Sociedad Argentina de Diabetes y de nuestro Comité, tu trabajo en el Hospital San Roque de San Salvador de Jujuy, tu logro académico y profesional que implica tanto sacrificio, pero especialmente por haber sido una gran persona, siempre atento con actividades académicas, presentaciones en Congresos hasta éste, tu último trabajo, pero lo más importante, tu dedicación a la comunidad. Desde el Comité de Pie Diabético siempre estarás presente. Hasta siempre amigo y colega.

\section{Agradecimientos}

Agradecemos la colaboración de las Dras. Valeria Landivar, Mónica Cusme Correa, Fernanda Castillo Zurita y Florencia Carballo, y de los Dres. Roger Vía y Daniel Pontelli en la realización del presente trabajo.

\section{BIBLIOGRAFÍA}

1. Boulton A. The diabetic foot: grand overview, epidemiology and pathogenesis. Diabetes Metab Res Rev 2008; 24:S3-S6.

2. Rodríguez-Bolaños RA, Reynales-Shigematsu LM, GiménezRuiz JA, et al. Costos directos de atención médica en pacientes con diabetes mellitus tipo 2: análisis de microcosteo. Rev Pan Salud Pub 2010; 28:412-420.

3. Hicks C, Selvin E. Epidemiology of peripheral neuropathy and lower extremity disease in diabetes. Curr Diab Rep 2019; 19(10):86

4. Ruiz M. Diabetes mellitus. Cuarta edición. Ed AKADIA. 2011.

5. Pop-Busui R, Boulton A, Feldman E, et al. Diabetic neuropathy: a position statement by the American Diabetes Association. Diabetes Care 2017; 40(1):136-154.

6. Hinchliffe R, Fortsythe R, Apelqvist J, et al. IWGDF guidelines on diagnosis, prognosis and management of peripheral arterial disease in patients with a foot ulcer and diabetes. Disponible en: https:// iwgdfguidelines.org/pad-guideline/. Consulta: octubre de 2020.

7. Gibbons G, Shaw P. Diabetic vascular disease: characteristics of vascular disease unique to the diabetic patient. Semin Vasc Surg 2012; 25:89-92.

8. Pedrosa H, Campillo N, Braver J, Antonucci R, JubizY, Barragan D. Consenso pie diabético ALAD-GEPLED. Revista de la Sociedad Argentina de Diabetes 2013; 47: 93-114.

9. Singh N, Armstrong DG, Lipsky BA. Preventing foot ulcers in patients with diabetes. JAMA 2005; 293:217-228.

10. Bus S, Lavery L, Monteiro-Soares M, Rasmussen A, Raspovic A, Sacco I, et al. IWGDF guidelines on the prevention and management of diabetic foot disease 2019. Disponible en: https:// iwgdfguidelines.org/wp-content/uploads/2019/05/02-IWGDFprevention-guideline-2019.pdf. Consulta: octubre de 2020.

11. Ibrahim A, Jude $E$, Langton $K$, et al. IDF clinical practice recommendations on the diabetic foot 2017. Disponible en: https://www.idf.org/ about-diabetes/54-our-activities/222-idf-clinical-practice-recommendations-on-the-diabetic-foot.html. Consulta: octubre de 2020.

12. Takahashi $O$, Sakakibara $R$, Tateno F, AibaY. Diabetic neuropathy: distribution pattern revisited. Case Rep Neurol 2020; 12:160-164.

13. Domínguez C, Flores C, Fuente G, et al. Actualización del tratamiento de la polineuropatía diabética periférica dolorosa 2015. Comité de Neuropatía Diabética. Sociedad Argentina de Diabetes. Revista de la Sociedad Argentina de Diabetes 2016; 50:35-46.

14. Moss SE, et al. The prevalence and incidence of lower extremity amputation in a diabetic population WESDR. Arch Intern Med 1992; 152:610-616.

15. Carro G, Saurral R, Salvador F, et al. Pie diabético en pacientes internados en Hospitales de Latinoamérica. Medicina (Buenos Aires) 2018; 78:243-251.

16. Miller J, Carter E, Shih J, et al. How to do a 3-minute diabetic foot exam. The Journal of Family Practice 2014; 63:646-656.

17. Dorresteijn J, Kriegsman D, Assendelft W, et al. Patient education for preventing diabetic foot ulceration. Cochrane Database Syst Rev 2014; (12):CD001488.

18. Sharma S, Kerry C, Atkins $H$, et al. The Ipswich touch test: a simple and novel method to screen patients with diabetes at home for increased risk of foot ulceration. Diabet Med 2014; 31(9):1100-1103. 